\title{
Publisher Correction: Organocatalysis in aqueous media
}

Michelle P. van der Helm, Benjamin Klemm and Rienk Eelkema (D)

Nature Reviews Chemistry (2019) https://doi.org/10.1038/s41570-019-0116-0

Published online 17 July 2019

In the main text, a citation to Ref 55 has been changed to Ref 182 (and vice versa). In the Supplementary information, corrections were made to reactions 16-18, and Refs 4, 5 and 42. Compounds numbers in the text have been styled to emphasize they represent chemical species.

https://doi.org/10.1038/s41570-019-0125-z I Published online 29 July 2019 